\title{
Research Paper on Java Interactional Development Environment Programming Tool
}

\author{
Prof. B.A. Jadhawar ${ }^{1}$, Komal A. Bhosale ${ }^{2}$ \\ Professor, Computer Science \& Engg, DACOE, Karad, India ${ }^{1}$ \\ Student, Computer Science \& Engg, DACOE, Karad, India ${ }^{2}$
}

\begin{abstract}
Java language is become very popular and research project deal with improvement of the language or its run time behaviour. A Java IDE (Integrated Development Environment) is a application which enables users to more easily write the code and debug Java programs. its also helpful for beginners. In this IDEs provide features like syntax highlighting and auto code completion, which help the user to code more easily. The IDE is a Free and Open Source IDE for software developers. The IDE runs on many platforms including Windows, GNU/Linux and Mac OS X. It is easy to install. You can easily create Java applications for mobile devices using Mobility Pack in JIDE. With the IDE has become one of the most preferred development tools, whether it be designing a Swing UI, an enterprise application or using it as a platform for creating your own IDE
\end{abstract}

Keywords: Integrated Development Environment (IDE), Hypertext Mark up Language (HTML), Abstract Window Toolkit (AWT), Java Virtual Machine (JVM).

\section{INTRODUCTION}

Now a days the largest source of information is World Wide Web. A lot of recent work has concentrated on earning knowledge from structured information on web. However, it is problematic how much valuable knowledge we can extract from lists and web tables. An even smaller percentage of them contain information understandable without context. In implementing computer-based information systems, specialized approach must be given to software support the user interface as we have seen various software like Net beans, Eclipse, Form Designer etc, which creates various GUI with readymade code. An Integrated Development Environment or development interactive environment is a software application. This IDE provides overall facilities to computer developer for develop software program. An IDE contain of a source code editor, build automation tools and a debugger. Most IDEs have intelligent code completion facility. As Net beans helps to design the Graphical Design Interface with creation of code automatically at background. Its providing a JIDE which is itself a java program. It provides you the flexibility to produce the graphical view which is auto generated. Also it is providing mechanism for automatic creation of HTML pages to just select type of objects and specify the values of attributes. The Java is target and interpreted programming language with a small and easy-to-understand set of instructions (the byte code) programmers can implement and test their concepts in a very dignified way. The Java Organized Devolution scene programming tools is a very useful tool, used by Java applets and applications to help manage getting the right version of Java for a user's system. For programmers, it also provides a auto code generation. The interface automatically generates the HTML code.

\section{RELATED WORK}

Thus, the challenge in supporting event-driven software development is to provide tools and frameworks with zero runtime overhead or performance penalty.

$\square$ When design any java application give full code must be written in the text file then it has to be saved and run it from command prompt.

$\square$ There is a simple way to providing a JIDE which is itself a java program. It provides us the flexibility to produce the graphical view.

$\square$ In this graphical view can produce various types like Button, Frame, Text Field, Text Area, Checkbox, and Labels.

$\square$ It select the type of application i.e. Applet, Swing, HTML, or Editor; they can directly make any graphical look in that application.

$\square$ JIDE automatically provides the source code at the background which can be seen on code window.

$\square$ For HTML section the created file is saved as .html format, and we have to simply open it and saw the output.

IDE Tools: There are many IDE tools available for source code editor, built automation tools and debugger. Some of the tools are,

1. Eclipse

2. NETbeans

3. Code::Blocks

4. Code Lite

5. Dialog Blocks 
IARJSET

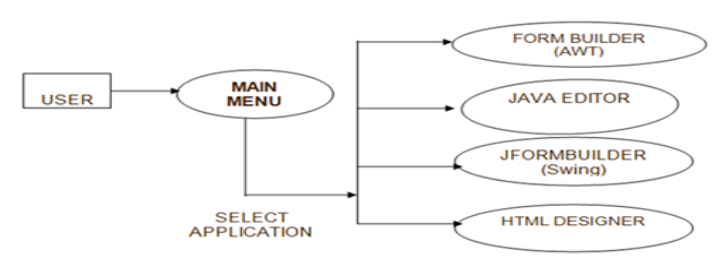

Fig. Architecture Diagram

\section{LITERATURE SURVEY}

An important decision making instructors of known programming courses is the choice of supporting software development tools. Usually this involves selecting an integrated development environment (IDE). Blue has received widespread adoption for first year courses that use the Java programming language [1].

Software security is a problem that has no easy solution. In day to day there are reports of companies being breached and personal data being compromised. Aim of this report is to search the problem of software security; this includes the development process of software and what is the role of security in that process.

This report discusses the design, implementation and testing of a product to help in developing secure software by integrating Static Code Analysis techniques into Integrated Development Environments (IDEs)[2].

\section{IMPLEMENTATION DETAILS}

Form Builder: In Form Builder we can create the applet forms with generation of the code at backend. We can specify each attribute of each AWT component specified in JAVA. AWT components such as Button, Frame, Text Field etc. We can adjust alignment and layouts of the AWT components required in forms.

Form builder is base tool they can create and generate specific code which users specified. which provide any exciting form with backend database and user friendly interface. To Manage manipulating your data from builder classes. AWT components (Abstract Windowing toolkit) java AWT component is an API To develop GUI or Window based application on java.

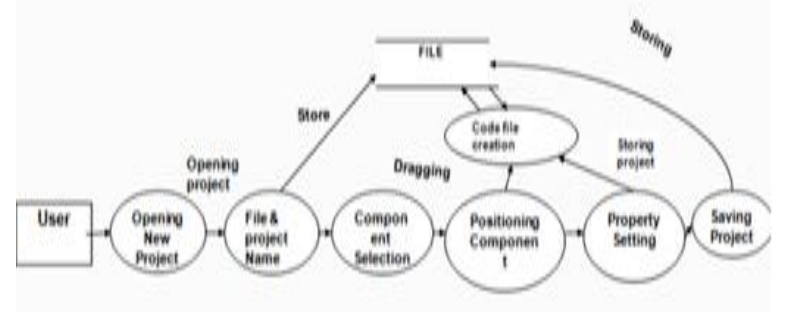

Fig. Form Builder

\section{Java Editor:}

In our editor we are providing the auto-completion mechanism. In which editor automatically shows the classes and methods supported by class of the respective object Java IDE is a software application which enables users to more easily write and debug java program. Many IDE's provide features like syntax highlighting, code completion, which help the user to code more easily.java editor provides specialized features for editing java code. Editor is a java specific outline view, which show the structure of active java completion unit. It is updated at the user edit the completion unit. Editor can also updated when the user edits the completion unit changes made in the code.

Editor Features-

1. Syntax highlighting

2.contain code assist

3. Quick fix

4. Integrated debugging features

5. Code formatting

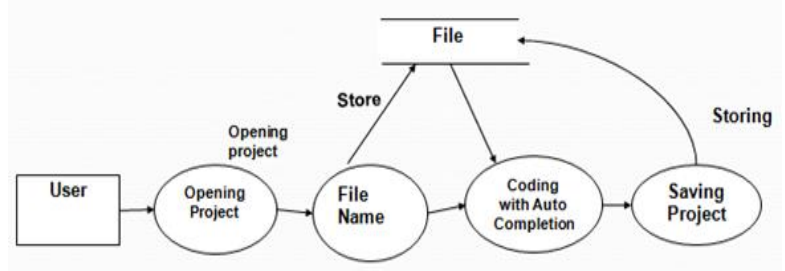

Fig. Java Editor

\section{JFormBuilder:}

In JFormBuilder can create the swing forms with generation of the code at backend. They can specify each attribute of each Swing component specified in JAVA. Swing components such as Button, JFrame, and JTextField etc. they can adjust alignment and AWT. Form Builder is the swing user interfaces which support for layout, group layout, table layout, grid layout, and make it easy. To design code of the programmer . Swing is GUI widget toolkit for java swing was developed to provide more sophisticated set of GUI component. Swing is set of program component of java programmers that provide the ability to create GUI component such as, Button, Scrollbar, that are independent of windowing System for specific OS.

Auto completion - In beginners level program they have faces more difficulty in coding so they are not understand where to complete the code completion helps the programmer where to complete class, method, fields, keyword, code completion supported file types and customs file types. In the Form builder programmer first create the file then opening the new project and give the appropriate name to that project then Program is store in the file. Then programmer selecting the component first debugging that component and positioning the appropriate component. 
IARJSET

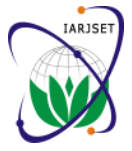

International Advanced Research Journal in Science, Engineering and Technology

National Conference on Innovative Applications and Research in Computer Science and Engineering (NCIARCSE-2017) AGTI's Dr. Daulatrao Aher College Engineering, Vidyanagar Extension, Karad

Vol. 4, Special Issue 4, January 2017

Programmer can set the property in that layout, margin set and save the file. And automatic generate code in the backend. That's JIDE feature helpful the beginners level Programmer.

Feature-

-Add fields with one click

-Drag and Drop recording.

-Multiple fields layout options arrange your fields in two, three, or mixture of columns.

\section{Designing simple HTML pages:}

We are providing mechanism for automatic creation of HTMLpages we have to just select type of objects and specify the values of attributes. In this word of internet era, everyone wants to create there own pages by using there own blocks, articles, photos, videos but some people choices there own way.

HTML - HTML is a hypertext makeup language is the Language use to create web pages everybody how is involve with the internet or web designing need to have basic understanding

HTML work or HTML code are return.HTML file Content" mark-up tags " that tell the browser how to follow instruction this tag.

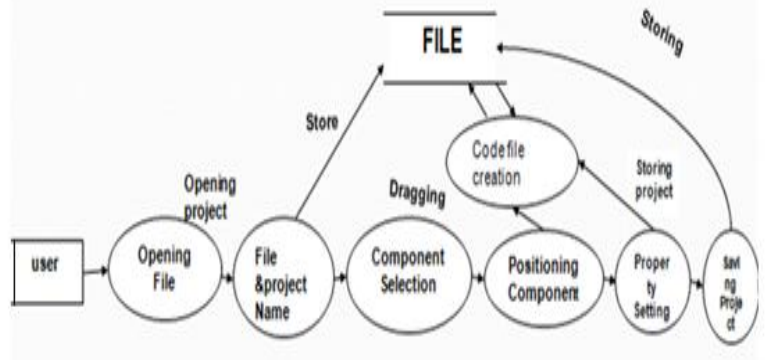

Fig.HTML Page

\section{Modules:}

We are providing mechanism for automatic creation of HTML pages we have to just select type of objects and specify the values of attributes.

To edit HTML code you only need a simple plane text editor, the rest depends on your skills and your creativity. However, you'll be more inspirited if you use the proper tools while you work.We know too that not everybody is able to pay for a licence copy of new code editor, that's when we use that open source. There are many free IDE's and code editor out there and you may want to know which may fit better to my requirement.

To make it easier for you or your team to develop web application, we have found some rarely good Integrated Development Environments for JavaScript, which provide you a convenient environment to code,edit,test, debug web application.

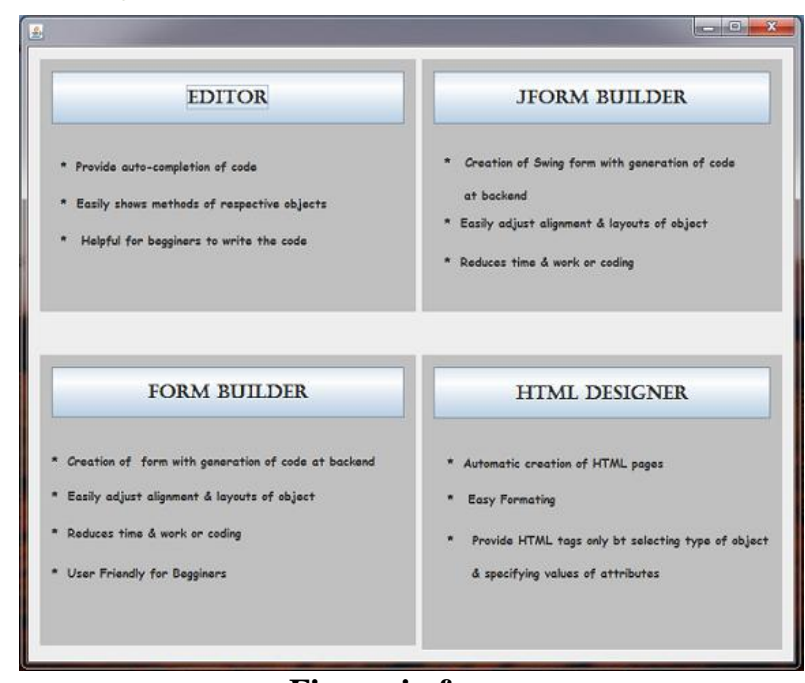

Fig. main frame

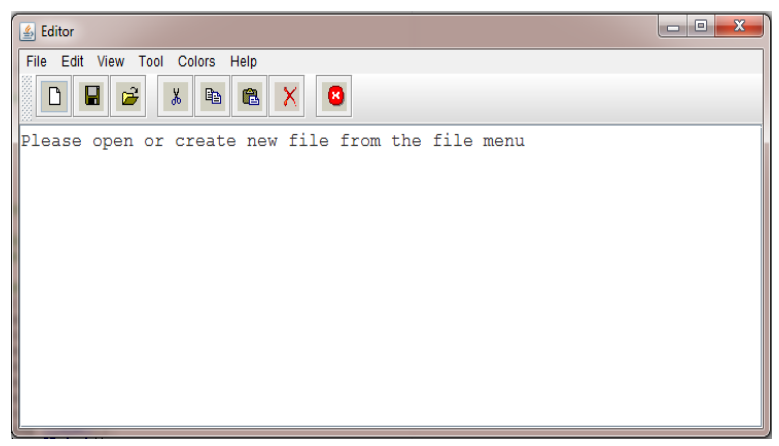

Fig. Editor

\section{CONCLUSION}

JIDE Tool is better over NETbeans for many reasons. Due to the complexity and extensibility of JIDE Tool, you will need additional resources to help you solve your specific problems. We have successfully developed IDE for easily coding, debugging, to easily understanding code at beginners programmer Compared to NETbeans, Eclipse they can compile programs faster and generates smaller executable and portable files in addition to friendly interface.

It provides a set of IDE for users to develop multimedia applications using C. Compared with NETbeans, Eclipse this is helpful to increase user's interest in JAVA programming. However, the experimental result shows that it is very important to improve the execution performance although it provides a User friendly interface for IDE, and high portability of executable files. We will address this issue in future.

\section{ACKNOWLEDGMENT}

We would like to acknowledge our gratitude to Prof. B. A. Jadhawar for valuable suggestions in carrying our research work. We also take opportunity to thank my friends for supporting me. 
IARJSET

\section{REFERENCES}

[1] Comparison of integrated development environment (ide) debugging tools: eclipse vs net beans. $* 1$ mrs. kavita s., $* 2 \mathrm{~ms}$. sindhu s.,(july2015).

[2] Dr. j vs. the bird: java ide's one-on-one* - Michael olan

[3] . Lee, "What's ahead for embedded software?," Computer, vol. 33,pp. 18-26, Sep 2000.

[4] Kölling, Michael and Bruce Quig, Andrew Patterson, John Rosenberg. "The BlueJ System and Its Pedagogy," Journal of Computer Science Education, Vol 13, No 4, December 2003.

[5] Stoler, Brian. "A Framework for Building Pedagogic Java Programming Environments", Master's Thesis, Rice University, April 2002.

[6] Kölling, Michael. "The Problem of Teaching Object- Oriented Programming, Part 2: Environments", Journal of Object-Oriented Programming, 11(9): 6-12, 1999

[7] H. Kopetz and G. Bauer, "The time-triggered architecture," Proceedings of the IEEE, vol. 91, pp. 112-126, Jan 2003.

[8] P. Levis, S. Madden, J. Polastre, R. Szewczyk, K. Whitehouse, A. Woo,D. Gay, J. Hill, M. Welsh, E. Brewer, et al., "Tinyos: An operating system for sensor networks," in Ambient intelligence, pp. 115-148,Springer, 2005.

[9] N. Burri, R. Flury, S. Nellen, B. Sigg, P. Sommer, and R. Wattenhofer,Yeti: an eclipse plug-in for tinyos 2.1," in Proceedings of the $7^{\text {th }}$ ACM Conference on Embedded Networked Sensor Systems, pp. 295-296, ACM, 2009.

[10] E. Cheong, E. A. Lee, and Y. Zhao, "Viptos : a graphical development and simulation environment for tinyos-based wireless sensor networks, “in SenSys, vol. 5, pp. 302-302, 2005.

\section{BIOGRAPHIES}

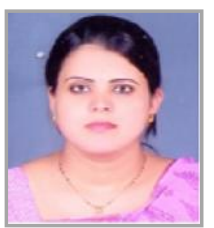

Prof. Jadhawar B. A. has master degree in Computer Science and Engineering from WCE,Sangaliin Shivaji University. She has 9.1 years of experience in teaching field. Currently working as Assistant Professor at DACOE, Karad. Her area of interest is database.

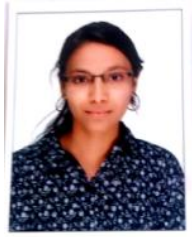

Ms. Bhosale Komal A. Currently doing Bachelor degree in computer engineering from Shivaji University at DACOE. 